
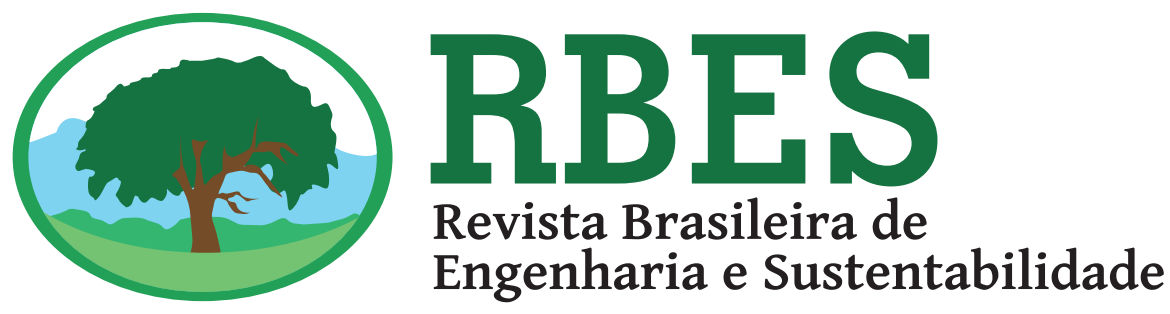

\title{
NANOCOMPÓSITO POLÍMERICO DE POLIAMIDA 6 REFORCADO COM ÓXIDO DE GRAFENO: PROCESSAMENTO VIA MISTURA EM SOLUC̣ÃO
}

MELO, C. C. N. ${ }^{1}$; MACHADO, F. M. ${ }^{2}$; OLIVEIRA, A. D. ${ }^{3}$; AVILA, E. S. ${ }^{4}$; RANGEL, E. M. ${ }^{5}$; CARVALHO, C. 0.6;

RODRIGUES, D. L. C 7 .

${ }^{1}$ Universidade Federal de Pelotas - Centro de Desenvolvimento Tecnológico/CDTec - Pelotas/RS, Mestrando em Ciência e Engenharia de Materiais - caio12cnmahotmail.com

¿Universidade Federal de Pelotas - Centro de Desenvolvimento Tecnológico/CDTec - Pelotas/RS, Docente do Programa de Pós Graduação em Ciência e Engenharia de Materiais/PPGCEM - fernando.machado.machado80Agmail.com

${ }^{3}$ Universidade Federal de Pelotas - Centro de Desenvolvimento Tecnológico/CDTec - Pelotas/RS, Docente do Programa de Pós Graduação em Ciência e Engenharia de Materiais/PPGCEM - amandaoliveira82lagmail.com

«Universidade Federal de Pelotas - Centro de Desenvolvimento Tecnológico/CDTec - Pelotas/RS, Graduanda em Engenharia de Materiais - elizetesouzadeavilalahotmail.com

5Universidade Federal de Pelotas - Centro de Desenvolvimento Tecnológico/CDTec - Pelotas/RS, Doutoranda em Ciência e Engenharia de Materiais - eduardamrangelagmail.com

UUniversidade Federal de Pelotas - Centro de Desenvolvimento Tecnológico/CDTec - Pelotas/RS, Mestranda em Ciência e Engenharia de Materiais - carolineoli.caragmail.com

${ }^{7}$ Universidade Federal de Pelotas - Centro de Desenvolvimento Tecnológico/CDTec - Pelotas/RS, Mestrando em Ciência e Engenharia de Materiais - daniel.l.c.rodriguesQagmail.com

\section{Palavras-chave:}

nanocompósito, reforço, grafeno, dispersão, náilon 6.

\begin{abstract}
Resumo
No presente estudo foram investigadas a síntese e caracterização de nanocompósitos de poliamida 6 reforçada com óxido de grafeno através da rota de mistura por solução. 0 óxido de grafeno foi sintetizado via método Hummers modificado, e o nanocompósito foi preparado em solução de ácido fórmico e posteriormente precipitado em água destilada. 0 material obtido foi então caracterizado para a avaliação da efetividade da metodologia empregada na dispersão do óxido de grafeno na matriz polimérica. As amostras foram caracterizadas através das técnicas de espectroscopia Raman, difração de raios $X$, termogravimetria e espectroscopia na região do infravermelho com transformada de Fourier. A rota de síntese do óxido de grafeno provou-se bastante eficiente, obtendo-se um material com alto grau de oxidação e esfoliação. Por meio dos resultados obtidos para o nanocompósito se pode comprovar a interação entre o reforço e a matriz polimérica, bem como as alterações estruturais de cristalinidade na poliamida 6 após a adição do óxido de grafeno. 0 nanocompósito produzido apresenta potencial aplicação como polímero de engenharia.
\end{abstract}

\section{GRAPHENE OXIDE-REINFORCED POLYAMIDE 6 POLYMER NANOCOMPOSITE: SOLVENT BLENDING PROCESSING}

Keywords: nanocomposite, reinforcement, graphene, dispersion, nylon 6.

\begin{abstract}
In the present study, the synthesis and characterization of graphene oxidereinforced polyamide 6 nanocomposites through the solution-mixing route was investigated. Graphene oxide was synthesized by the modified Hummers method, and the nanocomposite was prepared in formic acid solution and subsequently precipitated in distilled water. The material produced was then characterized for the evaluation of the effectiveness of the methodology used in the dispersion
\end{abstract}


of the graphene oxide in the polymer matrix. The material was characterized by the techniques of Raman spectroscopy, X-ray diffraction, thermogravimetry and Fourier transform infrared spectroscopies. The route of synthesis of the graphene oxide proved to be very efficient, obtaining a material with high degree of oxidation and exfoliation. By means of the characterizations of the nanocomposite, the interaction between the reinforcement and the polymeric matrix, as well as the structural changes of crystallinity in the polyamide 6 after the addition of the graphene oxide can be proved. The nanocomposite produced has potential application as an engineering polymer.

\section{INTRODUÇÃO}

Um material pode ser classificado como um nanocompósito quando pelo menos uma das fases constituintes tem uma dimensão inferior a $100 \mathrm{~nm}$ (AJAYAN et al., 2003). Ao contrário dos materiais compósitos tradicionais, que utilizam percentuais bastante elevados de reforço para que o desempenho do material final seja apreciável, os nanocompósitos são materiais que apresentam um percentual de reforço bastante reduzido (MARINUCCI, 2011). Essa característica é alcançada devido a elevada área de contato e/ou razão de aspecto dos reforços nanométricos (MITTAL et al., 2015).

Dentre as diferentes classes de materiais poliméricos utilizados atualmente como matriz, destaca-se a poliamida 6, também conhecida como náilon 6 (PA6). Esse termoplástico, caracterizado pela boa processabilidade, resistência química e qualidade elevada de acabamento superficial, tem sido bastante utilizado como polímero de engenharia (FU et al., 2015). Porém, para muitas aplicaçóes, tal material não apresenta propriedades mecânicas e estruturais satisfatórias. Por esse motivo, muito tem se estudado a fim de ampliar a aplicabilidade desse polímero, sobretudo na forma de nanocompósitos poliméricos (NCPs) (MOTOVILIN et al., 2011). Atualmente, nanoestruturas a base de carbono como, por exemplo, o óxido de grafeno (GO) têm sido propostas como promissores reforços poliméricos (ZHOU et al., 2015).

Através da inserção de GO como reforço de polímeros, tem-se ganhos em resistência mecânica e outras propriedades de engenharia ( $\mathrm{LOH}$ et al., 2010; MITTAL et al., 2015; TAN; PARK, 2015).
Porém, uma das barreiras para a aplicação desse nanomaterial na produçáo de nanocompósitos esbarra na tendência das nanopartículas à se aglomerarem, formando grandes clusters de reforço dispersos na matriz (NGUYEN et al., 2014). Portanto, o estudo da qualidade da dispersão e distribuição do reforço pela matriz, a interação dos componentes na interface e suas especificidades ainda estão em evoluçâo.

Diante deste contexto, o presente trabalho teve como objetivo sintetizar e caracterizar nanocompósitos de PA6/GO processado via mistura por solução. Esta técnica de processamento se apresenta bastante interessante por não necessitar de maquinário complexo e de custo elevado, além de se obter nanocompósitos com bom grau de distribuição e dispersão.

\section{MATERIAIS E MÉTODOS}

Foi utilizado neste trabalho o grafite em pó puro (Labsynth), PA6 comercial B300, fornecida pela Polyform, com densidade de $1,13 \mathrm{~g} / \mathrm{cm}^{3}$ e índice de fluidez de $2,9 \mathrm{~g} / 10 \mathrm{~min}\left(235^{\circ} \mathrm{C} / 2,16 \mathrm{~kg}\right)$, ácido sulfúrico $\left(\mathrm{H}_{2} \mathrm{SO}_{4}\right)$ PA (Dinâmica), nitrato de sódio $\left(\mathrm{NaNO}_{3}\right.$, Vetec), permanganato de potássio $\left(\mathrm{KMnO}_{4}\right.$, Labsynth), peróxido de hidrogênio $30 \% \quad\left(\mathrm{H}_{2} \mathrm{O}_{2}\right.$, Labsynth), ácido clorídrico ( $\mathrm{HCl}, \mathrm{Vetec})$, ácido fórmico $85 \%$ (Labsynth). Todos os reagentes foram utilizados sem purificação adicional.

\section{Obtenção do GO}

GO foi preparado segundo o método de Hummers modificado (JAURIS et al., 2016). Para esse procedimento 23,0 $\mathrm{mL}$ de $\mathrm{H}_{2} \mathrm{SO}_{4}$ e $0,5 \mathrm{~g}$ de $\mathrm{NaNO}_{3}$ foram adicionados a $1,0 \mathrm{~g}$ de grafite em pó. A mistura foi mantida em banho de água e sob forte agitação magnética por $10 \mathrm{~min}$. Após, 3,0 g de $\mathrm{KMnO}_{4}$ foram lentamente adicionados e a mistura permaneceu sob forte agitação magnética por $30 \mathrm{~min}$ (tempo de oxidação). Em seguida, 46,0 mL de água deionizada foram adicionados gota a gota para evitar que a temperatura ultrapasse $90{ }^{\circ} \mathrm{C}$. A mistura foi mantida em agitação por 15 min e então, $10,0 \mathrm{~mL}$ de $\mathrm{H}_{2} \mathrm{O}_{2}$ e 140,0 $\mathrm{mL}$ de água deionizada foram adicionados. $\mathrm{O}$ material sólido resultante, o GO, foi filtrado, lavado com $250,0 \mathrm{~mL}$ de uma solução de $\mathrm{HCl}$ e finalmente lavado com água destilada inúmeras vezes até que se atinja $\mathrm{pH}$ neutro. 


\section{Síntese do NCP}

O NCP a base de PA6/GO foi sintetizado através do método de mistura por solução (GONG et al., 2015). Para tanto, $70 \mathrm{~g}$ de PA6 foram solubilizadas em $200 \mathrm{~mL}$ de ácido fórmico sob agitação mecânica por duas horas a $70{ }^{\circ} \mathrm{C}$. Em seguida, 3,5 g de GO (5\% em massa de PA6) foram dispersos em ácido fórmico em ultrassom de ponta por $10 \mathrm{~min}$. O GO foi então adicionado ao polímero solubilizado, sob agitação constante. Para provocar a precipitação do NCP, 2 L de água destilada foram adicionados gota a gota ao sistema em agitação. $\mathrm{O}$ material precipitado (PA6/GO) foi posteriormente filtrado e lavado até $\mathrm{pH}$ neutro, mantido em agitação magnética em água a $70{ }^{\circ} \mathrm{C}$ para a remoção de ácido fórmico residual, novamente filtrado e seco em estufa por $24 \mathrm{~h}$ a $70{ }^{\circ} \mathrm{C}$.

\section{Caracterização dos materiais precursores: G0 e PA6}

Para avaliar as características dos materiais precursores do NCP, ou seja, o GO obtido via método Hummers modificado e a PA6, os materiais foram caracterizados por difração de raios X. Para tanto, utilizou-se um difratômetro BRUKER, modelo D2 PHASER, equipado com tubo de cobre (radiação com $\lambda=1.5418 \AA$ ), operando a $40 \mathrm{kV}$ e $40 \mathrm{~mA}$ com os seguintes parâmetros de ensaio: intervalo angular $5^{\circ}<2 \theta<35^{\circ}$, com passos de $0,05^{\circ}$ por um tempo de $1 \mathrm{~s}$ para cada passo. Ainda, os precursores foram analisados através da técnica de Espectroscopia Raman, em um equipamento Renishaw modelo InVia, utilizando uma linha de excitação de $\operatorname{Ar}(\lambda=532 \mathrm{~nm})$. Para se avaliar o comportamento do GO durante o aquecimento, realizou-se uma análise termogravimétrica (TGA) com o auxílio de termobalança Harrop STA-726 de $25^{\circ} \mathrm{C}$ até $1000{ }^{\circ} \mathrm{C}$ a uma taxa de aquecimento de $10{ }^{\circ} \mathrm{C} \mathrm{min}$ ${ }^{1}$. Espectroscopia no infravermelho por transformada de Fourier (FTIR) em um equipamento Shimadzu, IR Prestige21 também foi utilizada para a caracterização do GO, bem como da PA6.

\section{Caracterização do nanocompósito PA6/G0}

O nanocompósito PA6/GO sintetizado via o método de mistura por solução foi caracterizado através das técnicas de espectroscopia Raman, DRX e
FTIR. Os parâmetros utilizados nessas análises são os mesmos descritos anteriormente. Ainda, a morfologia do NCP obtido foi analisada com o auxílio de microscopia eletrônica de varredura (MEV), em um equipamento Shimadzu modelo SSX-550.

\section{RESULTADOS E DISCUSSÃO}

A espectroscopia Raman é uma técnica bastante útil na caracterização de materiais da família do carbono (YOUNG et al., 2012). Os materiais carbonosos apresentam dois picos comuns $\mathrm{G}\left(-1580 \mathrm{~cm}^{-1}\right)$ e $2 \mathrm{D}$ $\left(-2700 \mathrm{~cm}^{-1}\right)$ que são característicos do estiramento dos átomos $\mathrm{sp}^{2}$ na estrutura hexagonal do grafeno e a segunda ordem da banda D, respectivamente. A Figura 1A apresenta o espectro Raman do grafite precursor e também do GO obtido. Pode-se notar que uma banda pouco intensa aparece em $1350 \mathrm{~cm}^{-1}$ (banda D) para o grafite e é característica da presença de carbono $\mathrm{sp}^{3}$ e defeitos na estrutura e bordas do material (KUDIN et al., 2008). Sendo o grafite precursor um material em sua maioria cristalino, essa banda é quase inexistente. Nota-se, contudo, a elevação dessa banda, assim como o alargamento e deslocamento da banda G para o GO. Isto comprova a oxidaçâo das lamelas de grafite (KUDIN et al., 2008). Ainda podese observar a presença de uma banda pouco intensa em aproximadamente $1400 \mathrm{~cm}^{-1}$, característica da combinação da ressonância entre a banda $\mathrm{D}$ e bandas de defeitos quando medidas sob luz visível (FERRARI; BASKO, 2013).

Conforme já evidenciado na literatura (KUDIN et al., 2008), a intensidade da banda $D$ no espectro do GO aumenta enquanto a banda G sofre um alargamento. Isso é justificado pelo aumento da fração de átomos $\mathrm{sp}^{3}$ (defeitos e vacâncias) originados no processo oxidativo e de esfoliação. A razão entre as intensidades dessas bandas $\mathrm{D}$ e $\mathrm{G}\left(\mathrm{I}_{\mathrm{D}} / \mathrm{I}_{\mathrm{G}}\right)$ para o grafite em pó foi de aproximadamente 0,09 e para o material após oxidação o valor foi de 0,94 . Observase, portanto, o aumento do número de defeitos provocados pela oxidação, assim obtendo-se GO com maior grau de oxidação e abertura lamelar.

É claramente observado (Figura 1B) a presença das bandas D e G referentes ao GO na amostra de PA6/ GO, e apresentam-se mais largas e com uma razão 
$\mathrm{I}_{\mathrm{D}} / \mathrm{I}_{\mathrm{G}}$ para o NCP de 1,01 . Este valor é maior que o calculado para o GO puro e confirma a formaçáo de ligaçôes $\mathrm{sp}^{3}$ nos átomos de $\mathrm{C}$ da estrutura desse precursor. Isso ocorre pela funcionalização covalente das folhas de GO por cadeias de PA6 (PANT et al., 2012; DING, P et al., 2014). A banda em $-1447 \mathrm{~cm}^{-1}$ referente ao espectro da PA6 e PA6/GO, atribuída a conformação trans do grupo amida, corresponde a transformação cristalina da PA6 da fase $\gamma$ para a fase $\alpha$ (PANT et al., 2011).

$$
\text { A. }
$$

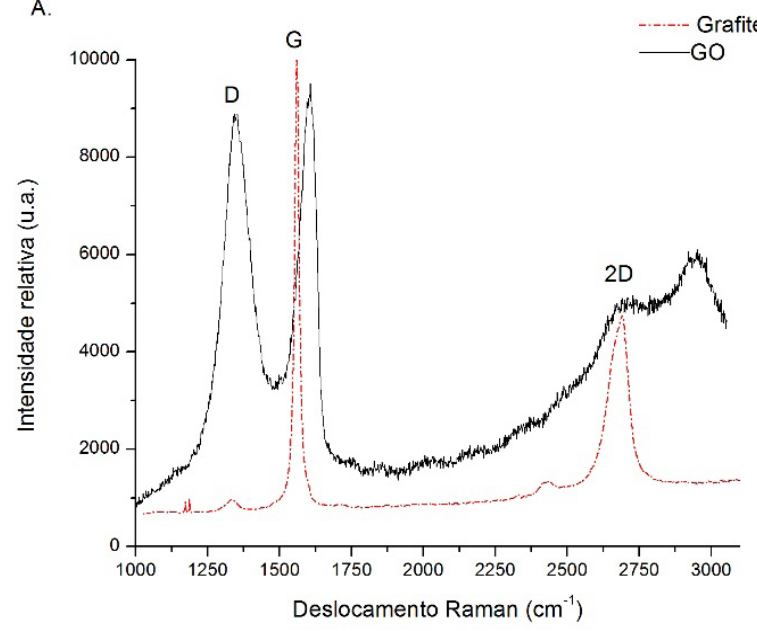

B.

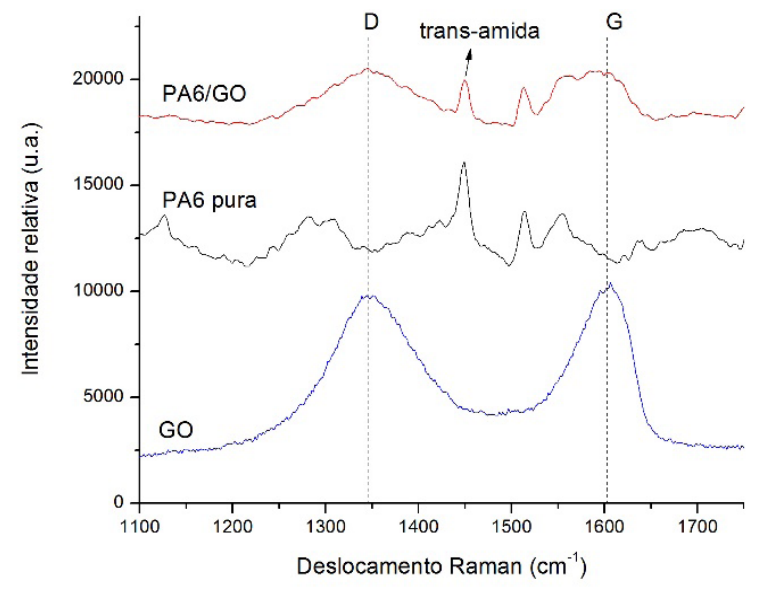

Figura 1 - A. Espectrograma Raman das amostras de grafite precursor e GO sintetizado. B. Espectrograma Raman das amostras de PA6 pura e PA6/G0.

Os resultados da análise termogravimétrica do grafite precursor e GO são apresentados na Figura 2. Observa-se que o grafite apresenta estabilidade térmica até aproximadamente $700{ }^{\circ} \mathrm{C}$. É observado também um ganho de massa de 1,07\% durante o aquecimento, que pode ser justificado por uma provável oxidaçáo do material em contato com fluxo de gás durante a análise. $\mathrm{O}$ comportamento térmico do GO se divide em três etapas: primeiro até temperaturas por volta de
$100{ }^{\circ} \mathrm{C}$ ocorre a remoção de água absorvida no material (remanescente do processo de síntese); a segunda etapa entre $130-340{ }^{\circ} \mathrm{C}$ que está relacionada com à retirada dos grupos funcionais oxigenados (hidroxilas, carboxilas, carbonilas e epoxilas) presentes; e acima dos $450{ }^{\circ} \mathrm{C}$ inicia-se a combustão da estrutura carbônica da amostra (MEHL et al., 2014).

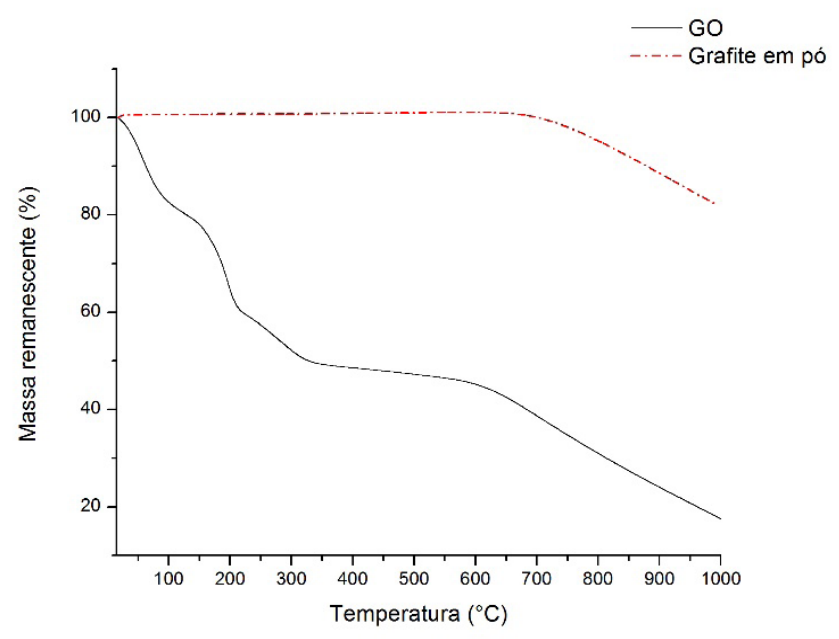

Figura 2 - Termograma das amostras de grafite precursor e GO.

A Figura 3 apresenta o espectrograma FTIR das amostras de GO, PA6 pura e PA6/GO. Observa-se para o GO (Figura 3A) a presença de uma banda bastante larga em torno de $3600 \mathrm{~cm}^{-1}$, que é atribuído ao grupo funcional hidroxila $(\mathrm{C}-\mathrm{OH})$, o que evidencia a hidrofilicidade do GO através da presença de umidade na amostra. Outros picos presentes correspondem aos grupos oxigenados originados no processo de oxidaçáa do grafite: $1716 \mathrm{~cm}^{-1}$ grupo carbonila e dupla ligação carboxílica $(\mathrm{C}=\mathrm{O}), 1587 \mathrm{~cm}^{-1}$ carbono aromático $(\mathrm{C}=\mathrm{C}), 1380 \mathrm{~cm}^{-1}$ estiramento no grupo carboxila $(\mathrm{OH}), 1030 \mathrm{~cm}^{-1}$ grupos alcóxi (XIONG et al., 2010; DING, PENG et al., 2014; O’NEILL et al., 2014).

Para a PA6 pura, é possível observar os picos característicos da sua estrutura polimérica. Em 1635 $\mathrm{cm}^{-1}$ apresenta-se o pico característico do estiramento da ligação $\mathrm{C}=\mathrm{O}$, em $1537 \mathrm{~cm}^{-1}$ o estiramento da ligação C-N (amida II), os picos em $2858 \mathrm{~cm}^{-1}$ e 2933 $\mathrm{cm}^{-1}$ são correspondentes ao estiramento da ligação $\mathrm{C}-\mathrm{H}$, o pico em $3296 \mathrm{~cm}^{-1}$ corresponde à ligação N-H (FU; ZHAO; et al., 2015). É possível observar as bandas características da fase $\alpha$ da PA6 nas bandas $930 \mathrm{~cm}^{-1}$ e $1200 \mathrm{~cm}^{-1}$ (Figura 2B), correspondentes à interação $\mathrm{CO}-\mathrm{NH}$ no plano cristalográfico da PA6 
(VASANTHAN; SALEM, 2001).

Para a amostra de PA6/GO, náo houveram grandes alteraçóes no espectrograma comparado ao da PA6 pura. Isso pode ser explicado pelo baixo percentual de GO adicionado ao polímero (5\% em massa). A figura $3 \mathrm{~B}$ apresenta um corte do espectrograma FTIR da PA6 e PA6/GO, onde nota-se uma redução de intensidade de todo o espectro do NCP e leve deslocamento da banda da amida II $\left(1537 \mathrm{~cm}^{-1}\right)$, o que demonstra a interação do GO com a PA6 através de ligaçôes de hidrogênio (FU; ZHAO; et al., 2015).
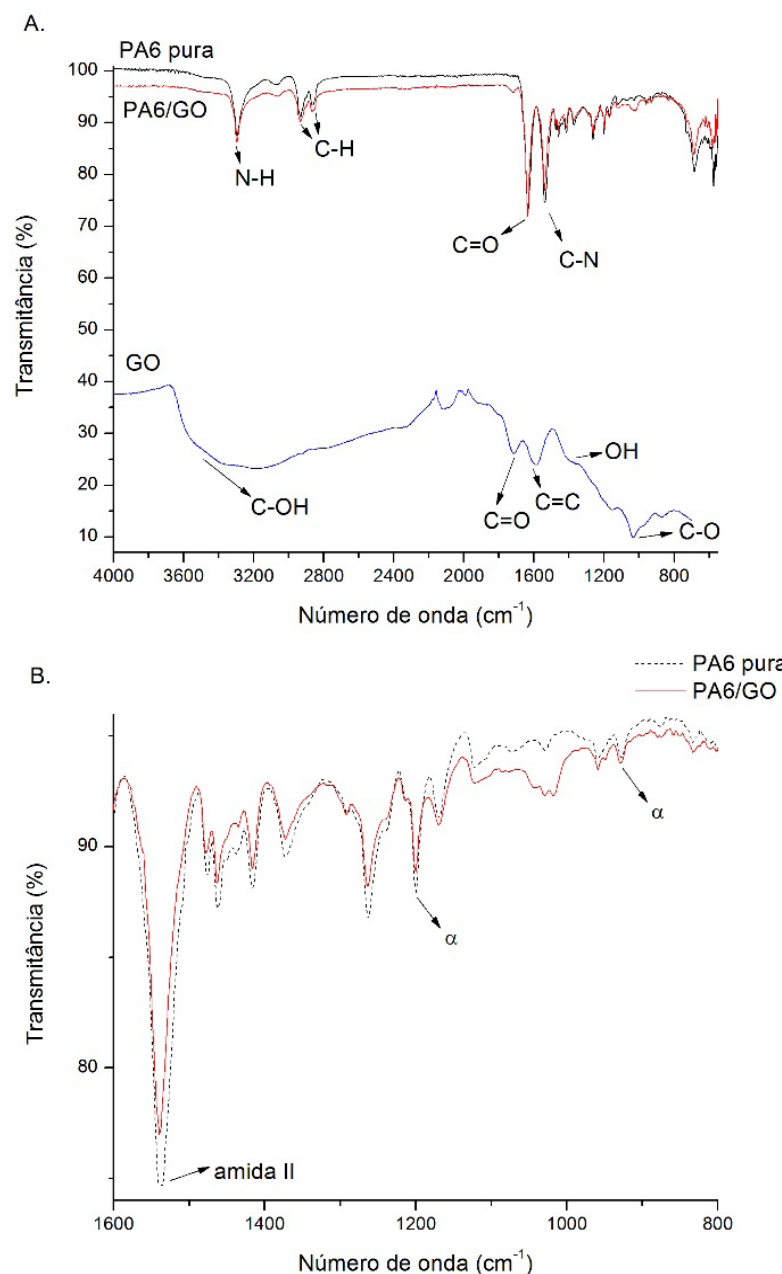

Figura 3 - A. Espectrogramas FTIR para G0, PA6 e PA6/G0, B. Corte nos espectrogramas da PA6 e PA6/GO.

A técnica de DRX (Figura 4) foi utilizada para corroborar as informações extraídas através da Espectroscopia Raman (ver Figura 1). Na Figura $4 \mathrm{~A}$ tem-se o difratograma do grafite precursor e do GO sintetizado. Já na Figura $4 \mathrm{~B}$ é apresentado o difratograma da PA6 pura e o nanocompósito PA6/ GO. A partir da Figura 4A, é possível observar um pico bastante intenso em aproximadamente $26,1^{\circ}$ que é característico do plano de difração (002) do grafite
(MEHL et al., 2014). Para o GO, observa-se que um máximo de difração em torno de $11^{\circ}$ está presente, com espaçamento interplanar calculado pela lei de Bragg de $\mathrm{d}(002)=0,864 \mathrm{~nm}$. Esse pico é atribuído ao conjunto
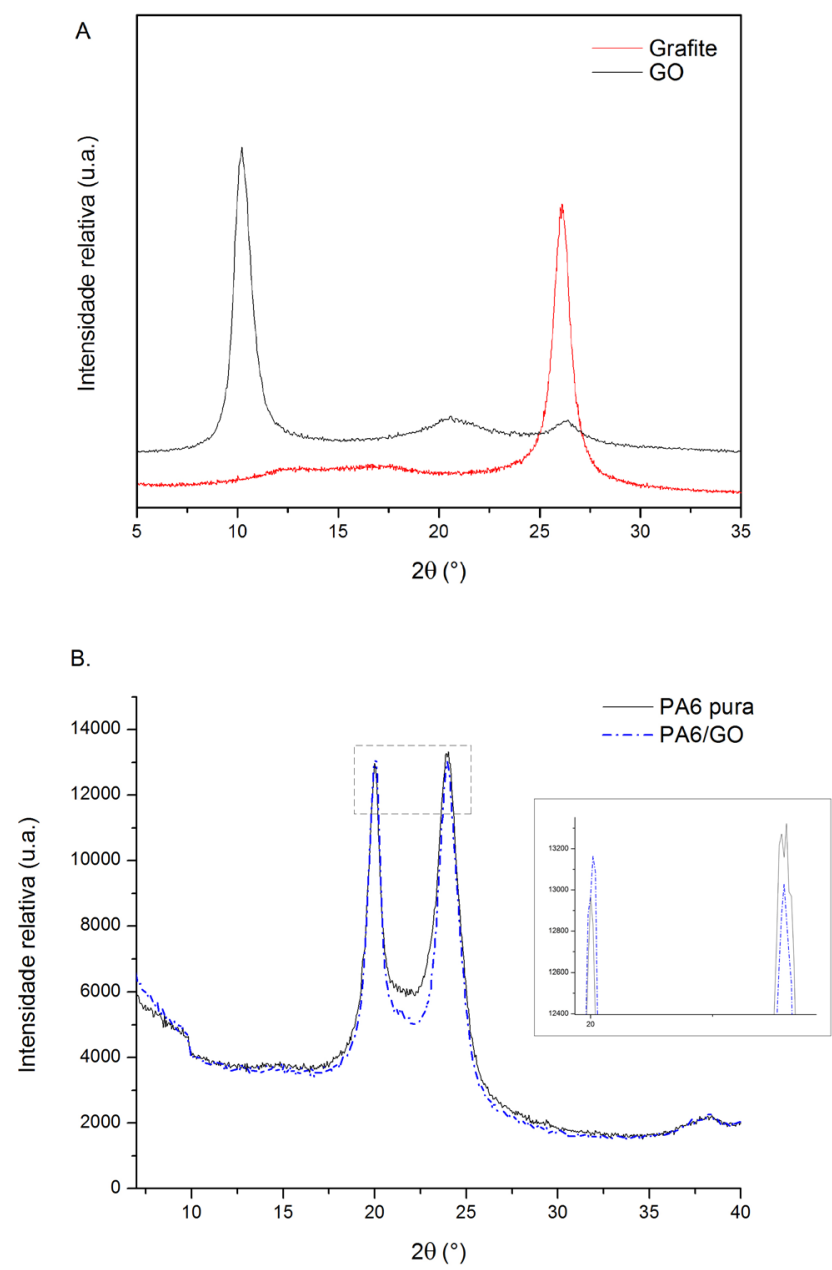

Figura 4 - A. Difratograma do grafite e $G 0$ e B. da PA6 e do nanocompósito PA6/GO.

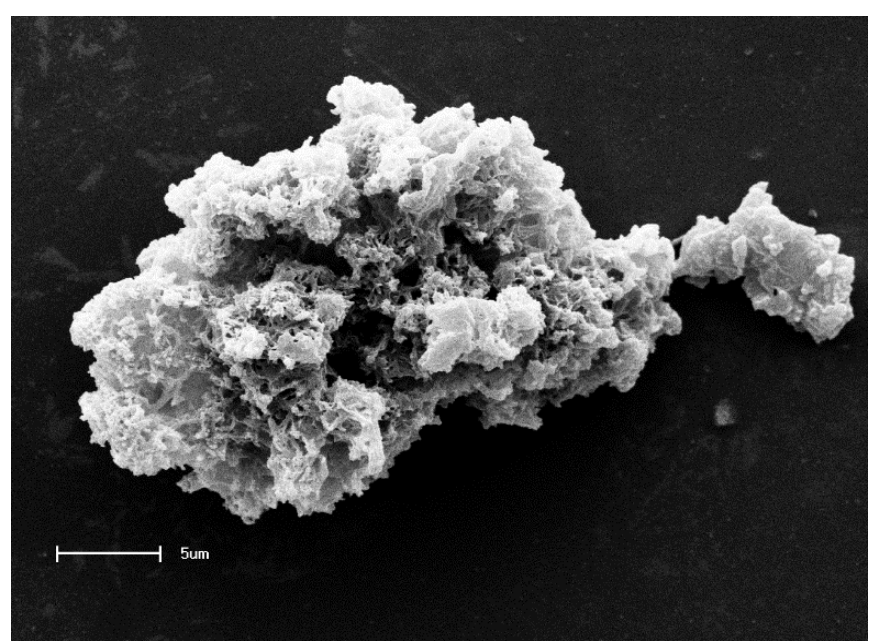

Figura 5 - Micrografia obtida via MEV da amostra de $\mathrm{PAb} / \mathrm{GO}$. 
de planos (001) do óxido de grafeno, relacionado à expansão das folhas do grafite devido à inserçáo de grupos oxigenados (BLANTON; MAJUMDAR, 2012). Um pico de baixa intensidade é observado em torno de $26,6^{\circ}$. Tal pico é bastante intenso no grafite, entretanto para a família do grafeno este pico deve (idealmente) desaparecer do difratograma, o que demonstraria uma completa esfoliação do material e com poucas camadas.

O difratograma da PA6 e do PA6/GO (Figura 4B) evidencia os planos de difração em $2 \theta=20^{\circ}\left(\alpha_{1}\right)$ e $2 \theta$ $=24^{\circ}\left(\alpha_{2}\right)$ da PA6 pura, e são referentes aos planos (200) e $(002,202)$ da fase cristalina $\alpha$ do polímero, respectivamente (DING, $\mathrm{P}$ et al., 2014). O máximo de difração em $11^{\circ}$ referente ao $\mathrm{GO}$ não aparece no NCP, o que pode significar um baixo empilhamento e ordenamento das lamelas do material, portanto apontando que o reforço está bem disperso e distribuído pela matriz (DING, PENG et al., 2014).

Observa-se também uma mudança de intensidade de $\alpha_{1}$ e $\alpha_{2}$ para o PA6/GO, quando comparado à PA6 pura. Esse comportamento indica que o comportamento cristalino do polímero foi afetado pela adição do reforço, ou seja, as ligações de hidrogênio entre as cadeias de PA6 sofreram perturbação na sua regularidade cristalina (FU; ZHAO; et al., 2015).

Através da análise por MEV (Figura 5), pode-se obter informaçôes a respeito da morfologia do NCP obtido. É possível observar que a amostra obtida a partir da precipitação por solução tem dimensóes micrométricas e sua forma é bastante irregular. Além disso, fica evidente a presença de poros. Devido a magnificação da imagem obtida náo se pode avaliar a dispersão e distribuição das lamelas de GO na matriz polimérica.

\section{CONCLUSÕES}

A síntese de GO através do método Hummers modificado provou-se bastante eficiente, obtendo-se um material com alto grau de oxidação e esfoliação. $\mathrm{O}$ método de mistura em solução é bastante viável para o preparo de nanocompósitos poliméricos. Através da técnica de difração de raio $\mathrm{X}$ pôde-se observar a alteração da estrutura cristalina da fase $\alpha$ da poliamida 6 com a inserção do reforço e também se comprova a interação entre GO e PA6 por formação de ligaçóes de hidrogênio evidentes nas análises por espectroscopia Raman e FTIR. O óxido de grafeno apresenta-se com baixo empilhamento e aglomeração. Através da técnica de microscopia eletrônica de varredura foi possível visualizar que o NCP obtido apresenta uma morfologia bastante irregular e porosa.

\section{AGRADECIMENTOS}

Agradecimentos ao Laboratório de Pesquisa em Materiais (LAPEM-UFPel) por ceder o espaço físico para a realização da parte experimental. À petroquímica Polyform Termoplásticos pela doaçáo da poliamida 6 utilizada neste estudo. Ao Centro de Microscopia Eletrônica do Sul (CEME-Sul-FURG), Laboratório de Materiais Cerâmicos (LACER-UFRGS) e Laboratório NovoNano (UFPel) pelas caracterizaçóes realizadas. Agradecimentos também a Capes, concessora da bolsa de mestrado da qual estrutura-se este trabalho.

\section{REFERÊNCIAS BIBLIOGRÁFICAS}

AJAYAN, P. M.; SCHADLER, L. S.; BRAUN, P. V. Polymerbased and Polymer-filled Nanocomposites. Nanocomposite Science and Technology. p.77-154, 2003.

BLANTON, T. N.; MAJUMDAR, D. X-ray diffraction characterization of polymer intercalated graphite oxide. Powder Diffraction, v. 27, n. 2, p. 104-107, 2012.

DING, P.; SU, S. S.; SONG, N.; et al. Influence on thermal conductivity of polyamide- 6 covalently-grafted graphene nanocomposites: varied grafting-structures by controllable macromolecular length. Rsc Advances, v. 4, n. 36, p. 18782 18791, 2014.

DING, P.; SU, S.; SONG, N.; et al. Highly thermal conductive composites with polyamide- 6 covalently-grafted graphene by an in situ polymerization and thermal reduction process. Carbon, v. 66, p. 576-584, 2014.

FERRARI, A. C.; BASKO, D. M. Raman spectroscopy as a versatile tool for studying the properties of graphene. Nat Nanotechnol, v. 8, n. 4, p. 235-246, 2013.

FU, X.; YAO, C.; YANG, G. Recent advances in graphene/ polyamide 6 composites: a review. RSC Adv., v. 5, n. 76, p. 61688-61702, 2015.

FU, X.; ZHAO, X.; YAN, D.; et al. A facile route to prepare fewlayer graphene/polyamide 6 nanocomposites by liquid reactive extrusion. RSC Adv., v. 5, n. 94, p. 77316-77323, 2015.

GONG, L.; YIN, B.; LI, L. P.; YANG, M. B. Nylon-6/Graphene composites modified through polymeric modification of graphene. Composites Part B: Engineering, v. 73, p. 49-56, 2015.

JAURIS, I. M.; MATOS, C. F; SAUCIER, C.; et al. Adsorption of sodium diclofenac on graphene: a combined experimental and theoretical study. Physical Chemistry Chemical Physics, v. 18, n. 3, p. 1526-1536, 2016. 
KUDIN, K. N.; OZBAS, B.; SCHNIEPP, H. C.; et al. Raman spectra of graphite oxide and functionalized graphene sheets. Nano Letters, v. 8, n. 1, p. 36-41, 2008.

LOH, K. P.; BAO, Q.; ANG, P. K.; YANG, J. The chemistry of graphene. Journal of Materials Chemistry, v. 20, n. 12, p. 2277, 2010.

MARINUCCI, G. Materiais Compósitos. Materiais Compósitos Poliméricos: Fundamentos e Tecnologia. $1^{\text {a }}$ ed., p.21-32, 2011.

MEHL, H.; MATOS, C. F.; NEIVA, E. G. C.; DOMINGUES, S. H.; ZARBIN, A. J. G. Efeito da variação de parâmetros reacionais na preparação de grafeno via oxidação e redução do grafite. Química Nova, v. 37, n. 10, p. 1639-1645, 2014. MITTAL, G.; DHAND, V.; RHEE, K. Y.; PARK, S.-J.; LEE, W. R. A review on carbon nanotubes and graphene as fillers in reinforced polymer nanocomposites. Journal of Industrial and Engineering Chemistry, v. 21, p. 11-25, 2015.

MOTOVILIN, M.; DENCHEV, Z.; DENCHEVA, N. On the Structure-Properties Relationship in Montmorillonite-Filled Polyamide 6 Nanocomposites. Journal of Applied Polymer Science, v. 120, p. 3304-3315, 2011.

NGUYEN, V. S.; ROUXEL, D.; VINCENT, B. Dispersion of nanoparticles: From organic solvents to polymer solutions. Ultrasonics Sonochemistry, v. 21, n. 1, p. 149-153, 2014.

O'NEILL, A.; BAKIRTZIS, D.; DIXON, D. Polyamide 6/ Graphene composites: The effect of in situ polymerisation on the structure and properties of graphene oxide and reduced graphene oxide. European Polymer Journal, v. 59, p. 353-362, 2014.
PANT, H. R.; BAEK, W. IL; NAM, K. T.; et al. Effect of lactic acid on polymer crystallization chain conformation and fiber morphology in an electrospun nylon-6 mat. Polymer, v. 52, n. 21, p. 4851-4856, 2011.

PANT, H. R.; PARK, C. H.; TIJING, L. D.; et al. Bimodal fiber diameter distributed graphene oxide/nylon-6 composite nanofibrous mats via electrospinning. Colloids and Surfaces A: Physicochemical and Engineering Aspects, v. 407, p. 121125, 2012.

TAN, H.; PARK, S. Y. Crystal structure evolution of nylon 6/ GOgraft nanocomposites during heat treatments and cold drawing. Polymer (United Kingdom), v. 78, p. 111-119, 2015. VASANTHAN, N.; SALEM, D. R. FTIR spectroscopic characterization of structural changes in polyamide- 6 fibers during annealing and drawing. Journal of Polymer Science: Part B, v. 49, p. 536-547, 2001.

XIONG, Z.; ZHANG, L. L.; MA, J.; ZHAO, X. S. Photocatalytic degradation of dyes over graphene-gold nanocomposites under visible light irradiation. Chemical Communications, v. 46, p. 6099-6101, 2010.

YOUNG, R. J.; KINLOCH, I. A.; GONG, L.; NOVOSELOV, K. S. The mechanics of graphene nanocomposites: A review. Composites Science and Technology, v. 72, n. 12, p. 14591476, 2012.

ZHOU, L.; LIU, H.; ZHANG, X. Graphene and carbon nanotubes for the synergistic reinforcement of polyamide 6 fibers. Journal of Materials Science, v. 50, n. 7, p. 2797-2805, 2015. 